\title{
Optical design of the 13 mega-pixels mobile phone camera
}

\author{
Pengbo Chen, Xingyu Gao ${ }^{\mathrm{a},{ }^{*}}$
}

Institute of Intelligent Opto-mechatronics and Advanced Manufacture Technology, College of Electromechanical Engineering, Guilin University of Electronic Technology, Guilin 541004,China.

a, ${ }^{*}$ gxy1981@guet.edu.cn

Corresponding Author: Xingyu Gao

Keywords: Optical design ; Mobile phone lens; Aspherical surface;

Abstract. According to the market demand for high resolution cell phone camera, a 13 mega-pixels camera lens applying aspherical surfaces is designed using the ZEMAX optical design software. The lens is consisted by four pieces of aspherical plastic lens, an optical filter and a piece of protection glass. The lens has the optical performance of F-number 2.8, viewing angle $76^{\circ}$, focal length $4.4 \mathrm{~mm}$, back focal length $0.58 \mathrm{~mm}$, and total length $5.6 \mathrm{~mm}$. After the tolerance analyzing, its tolerance meets the processing requirements, which can satisfy the requirements of the commercial sell phone.

\section{Introduction}

Since 2000, Japan's Sharp Co launched the world's first camera phone. Camera function has become an essential feature of the phone. In 2003, Sony launched the first mobile phone with camera function, model for Ericsson T618, this phone installed 10 million pixel mobile phone lens, which is the earliest a camera phone, marking China's entry into the era of mobile phone camera on the Chinese market. In 2006, Zhu Rihong [1], who designed a f / 3.2, field of view angle of 55 degrees, pixel reached 160 million of the lens, the lens system as the total length of $5 \mathrm{~mm}$.In 2008, Liu Maochao [2], who designed a F/2.85, field of view angle of 62 degrees, pixel to 3 million of the lens, the system length of the lens is 5.26mm.In 2009, Li Wenjing [3], who designed a F/2.8, the field of view angle of 65 degrees, pixel 5 million of the mobile phone lens, the total length of the lens system is $5.8 \mathrm{~mm}$. In 2011, Li Guang [4], who designed a F/2.45, field of view angle of 68 degrees, 8 million pixel mobile phone lens, the length of the lens is $7 \mathrm{~mm}$. Before the image sensor CMOS has not developed, it is very difficult to integrate the high pixel mobile phone lens in the mobile phone, and the length of the lens is generally more than $1 \mathrm{~cm}$. And now with the development of CMOS, its size is reduced, the size of the pixel from the previous 5um development to the present minimum can reach $1.1 \mathrm{um}$. Thus the total length of the mobile phone lens is much smaller than the total length of the previous lens. Also more conducive to the development of mobile phone structure light. In recent years, with the continuous upgrading of the function of the mobile phone optimization, people's mobile phone imaging results and the appearance of the phone is also increasing. Therefore, the design and production of high quality and low cost imaging and more compact mobile camera lens has become a hot spot. According to the above development form, the design is based on the selection of reasonable initial lens structure, the design of a 13 million pixel mobile phone camera.

\section{Design Points}

\subsection{The selection of image sensor CMOS}

In recent years, with the increasing perfection and maturity of CMOS processing technology, CMOS[5] imaging quality is getting better and better, and its performance parameters such as sensitivity, resolution, sensitivity, and so on have been greatly improved and improved. Because CMOS has the advantages of light weight, small size, low price, low power consumption, high integration, high read speed, etc. As a result, the vast majority of mobile phones on the market are 
using CMOS as a graphics processor. Now the production of CMOS manufacturers are mainly Omnivision, SONY, Samsung, Panavision, etc. The CMOS omnivision models for OV16880 CMOS sensor, its size is $8.3 \mathrm{~mm} 1 / 3.06 \mathrm{inch}$ and minimum pixel only for $1.12 \mathrm{um} \times 1.12 \mathrm{um}$, effective pixels $4224(\mathrm{H})$ x3136 (V), Image plane size for 4730um×3512um (diagonal 5.89mm). And support 1080p HD video recording at 60fps frame rate and at 30fps, 13 million pixels and full resolution $4 \mathrm{~K}$ ultra high definition video support.

Due to the presence of errors in the actual processing and assembly process will lead to surrounding the imaging appear dark corner, so in order to prevent the occurrence of such phenomena, design of semi high image must be greater than actual calculation of semi high image, the actual calculation of the half as high as $2.945 \mathrm{~mm}$. Therefore the design to $2.98 \mathrm{~mm}$. According to the principle of sampling and image sensor can display the maximum spatial frequency by Nyquist sampling frequency constraints, i.e. a spatially periodic at least 2 pixels, pixel size determines the resolution of the lens, they meet: $k=1 / 2 p$, where: $K$ for lens resolution, $P$ for the pixel size calculated the resolution of the lens is $446 \mathrm{lp} / \mathrm{mm}$, match the CMOS camera need spatial frequency analysis $446 \mathrm{lp} / \mathrm{mm}$.

\subsection{The main indicators of lens design}

According to the parameters of the CMOS image sensor and the actual needs of the camera. The design parameters of the mobile phone lens are shown in table 1.

Table 1 Design parameters

\begin{tabular}{lc}
\hline Parameter & Value \\
\hline Angle of view & $76^{\circ}$ \\
Aperture & 2.8 \\
Distortion & $2 \%$ \\
Focal length & $0.5 \mathrm{~mm}$ \\
Relative illumination & $50 \%$ \\
Optical length & $5.6 \mathrm{~mm}$ \\
Total pixels & $1300 \mathrm{~W}$ \\
\hline
\end{tabular}

\section{The Optical Design of Process}

\subsection{The lens initial structure selection}

To design an optical lens with good imaging quality, we should first select a suitable initial structure. If the initial structure selection is not reasonable, even if the optical design experience is very rich, it is difficult to achieve the desired results. Therefore, whether the initial structure is reasonable or not directly affects the final design results. There are many methods to choose the initial structure, and there are two methods in common use. The first is to calculate the initial structure by using the PW [6] method. This approach requires a wealth of knowledge of the theory of optical aberrations, the amount of computation is very large and designers have many years of design experience. The second is by finding relevant literature and patent invalid. Select a reasonable structure as the initial structure of the design, and then according to the design requirements of the selected initial structure for scaling. In the case of compliance with the design criteria, the structure is optimized. This design uses second methods to design optimization. As shown in Figure 1. Enter the selected initial structure in the ZEMAX software. The transfer function of the initial structure is shown in Figure 2. According to the transfer function curve shown in Figure 2, the image quality of the lens is very poor. In the full field of view, the value of MTF for $178 \mathrm{lp} / \mathrm{mm}$ is 0 . So this structure has a lot of optimization space. 


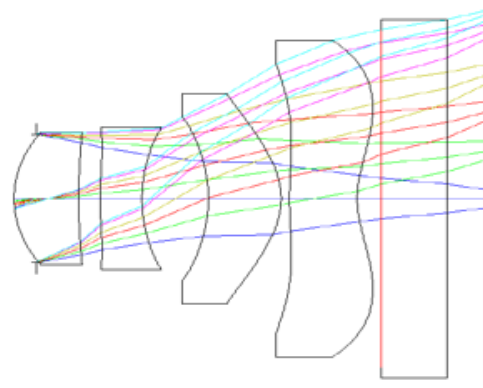

Fig.1 Intial structure

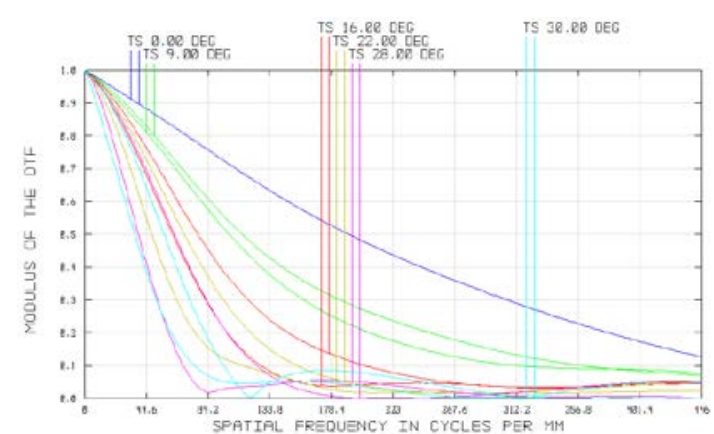

Fig.2 The initial structure of the MTF curve

\subsection{The choice of lens material}

According to statistics, in order to save costs, most of the mobile phone lenses are made of plastic materials and aspheric [7] lens. Optical plastic is a transparent non crystalline organic polymer, a single molecule polymerization, compared with the glass material, optical plastic with light quality, good light transmittance, low cost, easy to process and so on. The use of aspheric lens can effectively control the lens of a variety of aberrations, reduce the number of optical components, improve the system's relative aperture and field of view, and can also adjust the aspheric coefficient to improve optical performance. Because this design to achieve a wide angle of 76 degrees, so using the positive and negative combination of positive and negative, the structure of the form of $4 \mathrm{P}(\mathrm{P}$ said plastic lens). The first lenses is made of APL5514ML plastic material, this material has excellent transmittance, mobility, low birefringence, and the price is relatively low, so the use of large quantities as a mobile phone lens material. The second lenses using OKP4HT plastic material, this material for the plastic material in the high fold family, with a high refractive index and good molding effect, has been widely used as a mobile phone lens material. The third, four pieces of lens using model 480r plastic material.This material has low birefringence, low water absorption rate, high temperature resistance, is not easy to be attached to the electrostatic, look easy to maintain the characteristics, as mobile phone lens material has a high utilization rate. The fifth pieces for the K9 filter, the main filter out of the near infrared 700-1000nm.

\subsection{The optimization process}

According to the needs of the focal length of the system design requirements, the initial structure to zoom in, the focal length [8] scale is $4.5 \mathrm{~mm}$. When scaling to pay attention to the following points: the first point is the change of the boundary conditions, the second point is the concave lens center is too thin, the third point is not allowed to appear convex lens edge is too thin.

In order to meet the requirements of the actual processing, the optimization process of the lens thickness not set too small, to set the lens center and edge thickness is greater than $0.3 \mathrm{~mm}$. The radius, thickness, air gap, even sub surface coefficient and the two surface coefficient of each lens are all set as variables. Use the default evaluation function, and add the exact number of operations to optimize the limit, so that the efficiency of the optimization can be improved. The optimized operation number is shown as follows:

The total length of the TOTR control system is $5.6 \mathrm{~mm}$. Adding the DIMX operator to control the size of the distortion, the distortion is limited to $2 \%$. Add the dispersion of TRAC operand control imaging spot size. In order to make the lens and CMOS better coupling, the number of RAED control system to be added to control the main beam radiation angle, so that the output angle is less than 35 degrees. The MTF curve is expressed as the quality of the imaging quality, so it is necessary to add the number of operations MTFT and MTFS to optimize the MTF. After optimization, the structure of the mobile phone lens is shown in Figure 3. The field angle is 76 degrees, the system length is $5.6 \mathrm{~mm}$, the effective focal length is $4.4 \mathrm{~mm}$, the rear focal length is $0.56 \mathrm{~mm}$, the center and the edge thickness of all lenses are more than $0.3 \mathrm{~mm}$, which is in line with the actual processing requirements. 


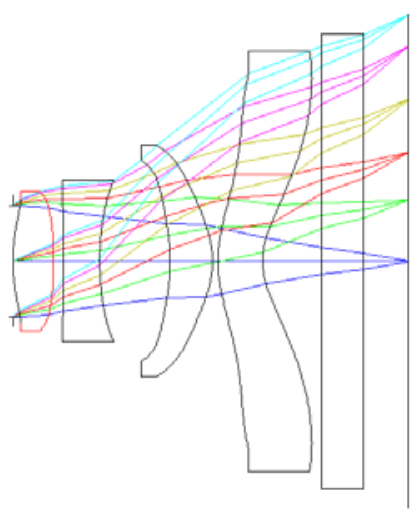

Fig.3 System structure after optimization

\section{Image Quality Analysis}

After optimization, the image quality has been greatly improved. MTF (modulation transfer function) is a function that can evaluate the imaging quality of optical system. The larger the space frequency represented by horizontal coordinates, the more the number of line pairs per millimeter can be resolved, the stronger the ability of resolving the details. It reflects the lens of the object at different frequencies of the transfer capability, the higher the MTF [9], the better the quality of the image. Under normal circumstances, the edge field of view of the lens imaging quality allows a certain range of decline. While the 0.7 field of view is the main guarantee for the imaging quality of the lens, the MTF curve of this field determines the quality of the image. Figure 4 shows the MTF curve under different field of view. After optimization, the center field of view 224lp/mm in MTF is greater than 0.5. In 446lp / $\mathrm{mm}$ is greater than 0.15 . It can be seen at a frequency of $224 \mathrm{lp} / \mathrm{mm}$, in addition to the edge of the visual field, the other field of view of the MTF value is greater than 0.25. In the 0.7 field of view, the value of MTF in the sagittal direction of $224 \mathrm{lp} / \mathrm{mm}$ was greater than 0.4 , the meridional direction is greater than 0.27. In the 0.7 field of view, the value of MTF in the sagittal direction of $446 \mathrm{lp} / \mathrm{mm}$ was greater than 0.09 , the meridional direction is greater than 0.03 . Although there are some astigmatism, but because of the small proportion so does not affect the overall picture. It shows that the imaging quality of the mobile phone lens is better, and it meets the requirement of imaging. According to figure 5 shows that the spot like spot on the surface of RMS is very small. It is mainly distributed in the airy 6.02um the size of the area, close to the diffraction limit [10] of imaging, meet the design requirements. Because the maximum field angle is larger than 76 degree, the field curve and distortion can not be too large. In Figure 6, the left represents the size of the mobile phone lens curvature of field, the right indicates the size of lens distortion. From left, meridional field curvature of the lens ( $\mathrm{T}$ curve) and loss of field curvature arc (S curve) are less than $0.15 \mathrm{~mm}$. From the right, the lens distortion control in the range of $2 \%$. The human eye can not feel the image distortion (distortion $<3 \%$ ), to meet the system design requirements. Figure 7 shows the relative illumination of the lens. The horizontal coordinates of the curve indicate the size of the field of view angle of the mobile phone, and the longitudinal coordinates indicate the relative illumination intensity. It can be known that the relative illumination of all field of view is more than 0.48 , which can satisfy the requirement of mobile phone lens design. 


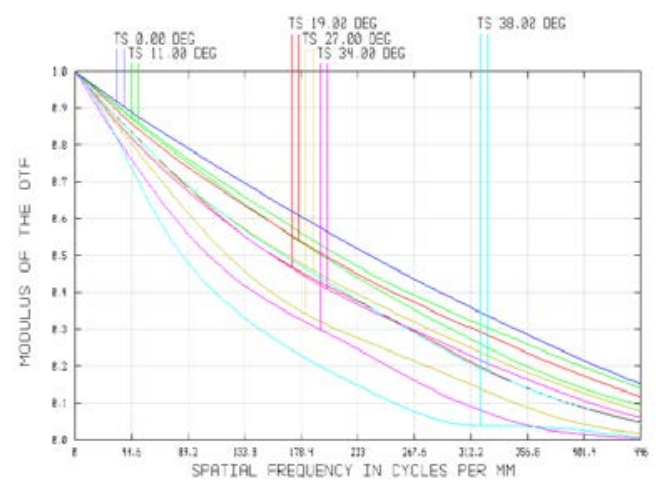

Fig.4 The optimized MTF curve
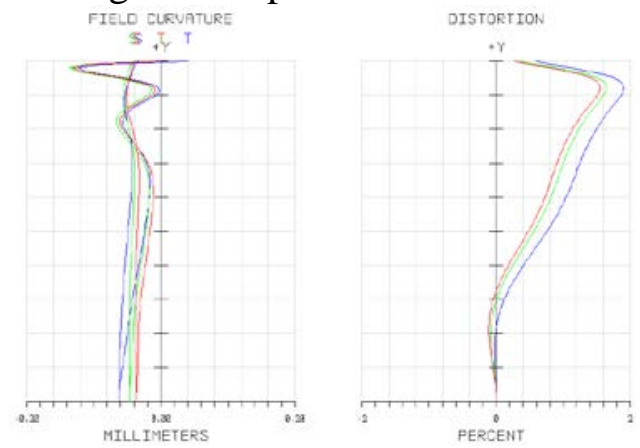

Fig.6 Field curvature and distortion
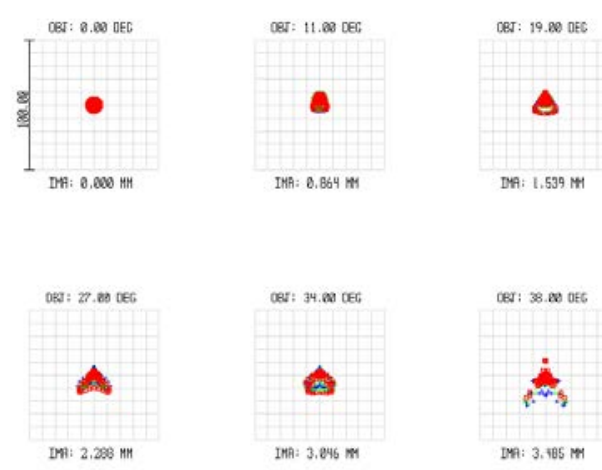

Fig.5 Spot diagram

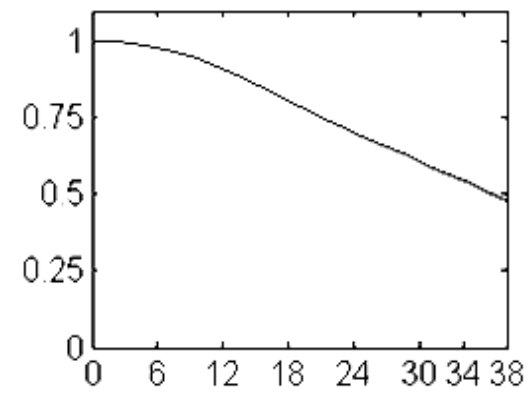

Fig.7 Relative illumination

\section{Tolerance Analysis}

A good design of the lens can be used in production and processing, in addition to a good image quality, but also must meet the existing processing level of error. Because if the system tolerance [11] too tight can cause processing costs, and even make the alignment process can not meet the requirements of the final image quality, it is necessary to carry out tolerance analysis. Using ZEMAX software to analyze the tolerance of the designed lens. Tolerance sensitivity analysis and Monte Carlo analysis are used to analyze the MTF value of the system as tolerance sensitivity. Finally get all the aspherical lens thickness tolerance of $\pm 0.01 \mathrm{~mm}$, IR cut filter thickness tolerance of $\pm 0.03 \mathrm{~mm}$, the radius of curvature of each surface tolerance of $0.01 \mathrm{~mm}$, the surface of each eccentric tolerance is $\pm 0.01 \mathrm{~mm}$, tilt tolerance of $\pm 0.3^{\circ}$, the refractive index of the lens material tolerance of \pm 0.002 , Abbe tolerance of \pm 0.5 , to meet the current level of processing.

\section{Conclusion}

In this paper, the initial structure is optimized by using ZEMAX optical software. In the end, a 13 million pixel mobile phone with a better imaging effect is obtained. The lens is characterized by its simple structure, better imaging effect, shorter total length of the optical system. Image surface of the angle of incidence of the principal ray of less than $30^{\circ}$, the lens and CMOS can guarantee a good coupling. In summary, the design of the lens to meet the actual needs of mobile phone production.

\section{Acknowledgment}

The work of this paper is supported by National Nature Science Foundation of China (Grant No.61265010) and Guangxi Natural Science Foundation (Grant No.2015jjBA70017).

\section{References}

[ 1 ] Zhu Hong, Wang Zhenhai , Zhang Wenxun. The high-quality cell phone camera design study -130 million mobile phone camera [D], Nanjing: Nanjing University of Technology, 2006 . 
[ 2 ] Liu Maochao, Zhang Lei ,Liu Peipei et al.300 megapixel camera phone [J]. Journal of Applied Optics , 2008,29 ( 6 ) : 944-948.

[ 3 ] Li Wenjing.500 megapixel camera phone optical design [J] Laser and Optoelectronics Progress , 2009 , 46 ( 1 ) : 56-59.

[ 4 ] Li Guang, Wang Jianye, Zhang Yan .800 megapixel camera phone of Design [J]. Journal of Applied Optics , 2011,03-0420-06.

[5] Xiao Zexin. Optical Design [M]. Beijing: Electronic Industry Press, 2002

[6] Li Xiaotong, Cen. Mega geometrical optics aberration optical design M]. Zhejiang: Zhejiang University press, 2007.

[7] Gou Zhiyong, Wang Jiang, Wang Chu, et al. Aspheric Optical Design Techniques [J]. Laser Journal, 2006,27 (3): 1-2.

[8] Hsin Yueh Sung, Sidney S, Horng Chang. Design of mobile phone lens with extended depth of field based on poing -spread function focus invariance[C]//SPIE, 2008, 7061: 706107-1-11.

[9] Yang Zhou, Yang Huiming, Ding Guilin. A slim 8 million pixel mobile phone lens design [J]. Application Optics, 2013, 34 (3): 413-419.413-419. (in Chinese)

[10]GEARY J M Introduction to lens design with practical zemax example [M].US:Willmann-bell,inc,2002.

[11] Ren Zhao-yu,Bai Jin-tao. Design of mobile phone camera lens based on ZEMAX.Appl.Opt.2010,31(1):34-38. 